\title{
Cold Highly Charged lons In A Penning Trap: Experiment and Simulation
}

J.P. Holder, L. Gruber, D. A. Church and D. H. Schneider

This article was submitted to

U. 5. Depgrtment of Enargy

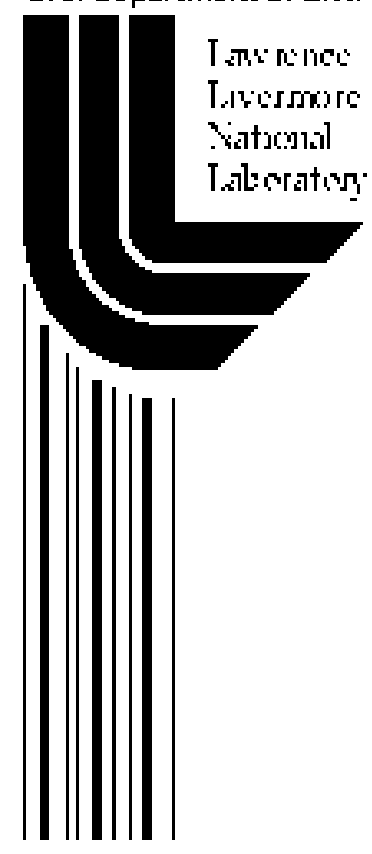

2001 Workshop on Non-Neutral Plasmas, San Diego, July 30 August 2, 2001.

August 18, 2001 


\section{DISCLAIMER}

This document was prepared as an account of work sponsored by an agency of the United States Government. Neither the United States Government nor the University of California nor any of their employees, makes any warranty, express or implied, or assumes any legal liability or responsibility for the accuracy, completeness, or usefulness of any information, apparatus, product, or process disclosed, or represents that its use would not infringe privately owned rights. Reference herein to any specific commercial product, process, or service by trade name, trademark, manufacturer, or otherwise, does not necessarily constitute or imply its endorsement, recommendation, or favoring by the United States Government or the University of California. The views and opinions of authors expressed herein do not necessarily state or reflect those of the United States Government or the University of California, and shall not be used for advertising or product endorsement purposes.

This is a preprint of a paper intended for publication in a journal or proceedings. Since changes may be made before publication, this preprint is made available with the understanding that it will not be cited or reproduced without the permission of the author.

This report has been reproduced directly from the best available copy.

Available to DOE and DOE contractors from the

Office of Scientific and Technical Information

P.O. Box 62, Oak Ridge, TN 37831

Prices available from (423) 576-8401

http:/ /apollo.osti.gov/bridge/

Available to the public from the

National Technical Information Service

U.S. Department of Commerce 5285 Port Royal Rd., Springfield, VA 22161

http://www.ntis.gov/

OR

Lawrence Livermore National Laboratory Technical Information Department's Digital Library http://www.llnl.gov/tid/Library.html 


\title{
Cold Highly Charged Ions In A Penning Trap: Experiment and Simulation
}

\author{
J. P. Holder ${ }^{+}$, L. Gruber* ${ }^{\star \S}$, D. A. Church ${ }^{+}$and D. Schneider* \\ ${ }^{+}$Physics Department, Texas A\&M University, College Station TX 77843-4242 \\ *Lawrence Livermore National Laboratory (LLNL), Livermore, CA 94550 \\ ${ }^{\S}$ Present Address: Spectra Physics Laser, Inc. 1305 Terra Bella Ave., Mountain View, CA 94043
}

\begin{abstract}
Using the LLNL EBIT/RETRAP system non-neutral plasmas of highly charged ions were produced and cooled to temperatures around one Kelvin. These strongly coupled plasmas can model white dwarf astrophysical plasmas in the laboratory. These systems may also have potential application to quantum computation. The experimental results from the last operations of the trap at Livermore are discussed. Molecular dynamics simulation results are discussed as a guide to past and future experiments. The status and future plans for RETRAP at LBNL's 88 inch Cyclotron are discussed.
\end{abstract}

\section{INTRODUCTION}

Strongly coupled plasmas are characterized by the ratio of the Coulomb interaction between particles with charge $q$ to the thermal energy of the particles $\left(\mathrm{k}_{\mathrm{B}} \mathrm{T}\right)$ being greater than one. This Coulomb coupling parameter:

$$
\Gamma=\frac{q^{2}}{4 \pi \varepsilon_{0} a_{0} k_{B} T}
$$

with the Wigner-Seitz radius

$$
a_{0}=\left(\frac{3}{4 \pi n}\right)^{\frac{1}{3}}
$$

Where $\mathrm{n}$ is the number density of the charged particles. If $\Gamma$ exceeds $\sim 172$ (for infinite one component plasma) the plasma crystallizes and is referred to as a "Coulomb crystal" or for finite crystals at higher Гs "Coulomb cluster"[1]. White Dwarf stars contain plasmas with fully stripped ions and degenerate electrons that can be treated as strongly coupled non-neutral plasmas. Crystallization and other thermodynamic properties (chemical layering, etc.) effect the luminosity function, which is used for astrophysical chronometry[2,3].

Cold, confined and relatively numerous highly charged ions are of interest for precision fundamental spectroscopic measurements[4]. These transitions, coupled with the charge scaling of motions in a trap, provide an additional "parameter space" for the investigation of trap based quantum coherence and computation experiments. To study mixed strongly coupled non-neutral plasmas and to prepare for precision fine-structure and hyperfine-structure laser spectroscopy on ground term transitions of highlycharged ions, a source of highly charged ions (EBIT) and a cryogenic Penning trap for capturing and cooling these highly charged ions (RETRAP) have been developed at Lawrence Livermore National Lab $[5,6,7]$. 


\section{EXPERIMENT}

The scheme used in the experiment to cool highly charged ions to the lowest realizable temperature via sympathetic cooling with laser cooled ions. $\mathrm{Be}^{+}$ions are used for both their low mass to charge ratio $(\mathrm{m} / \mathrm{q}=9)$ and the reasonable cooling laser transition (reachable with commercial available internally doubled ring dye laser). $\mathrm{Be}^{+}$ions were produced in a metal vapor vacuum arc (MeVVA) source. The $\mathrm{Be}^{+}$ ions were analyzed by a 90 degree bending magnet (to eliminate other charge states and species produced in the MEVVA) and focused through a series of ion optics into a tube at a potential near the MEVVA extraction potential of approximately $7.5 \mathrm{keV}$. A high voltage switch lowers the potential of the tube to near ground, "de-accelerating" the $\mathrm{Be}^{+}$ions, so they can then relatively slowly pass into a hyperbolic trap where an auxiliary "capture" electrode is pulsed to confine the ions. These $\left(\sim 10^{5}\right)$ ions were initially cooled to near room temperature by tuning (via trap ring-endcap voltage) their axial oscillation frequency into resonance with a cryogenic parallel tuned circuit. The heating of the parallel circuit by currents induced by fluctuations in the centroid of the confined ion motion, together with ionion collisional coupling, initially cools the ions at an exponential rate with a time constant of several minutes.

Eventually, the cooling is dominated by a transverse laser beam de-tuned several $\mathrm{GHz}$ below the cycling resonance transition near $313 \mathrm{~nm}$ [8]. With decreased de-tuning of the cooling laser, the ions then rapidly cooled to below $1 \mathrm{~K}$, as evidenced by a decrease in light scattering, and confirmed by probing the Be ions with a weak second laser tuned through a nearby non-cycling transition. Using slightly altered trap potentials more $\mathrm{Be}^{+}$ions can be 'stacked' into the trap, with a cooling rate enhanced by the presence of previously cooled Beryllium ions.

Then beam pulse of $\mathrm{Xe}^{44+}$ ions from the EBIT (analyzed by a similar magnet, passing through ion optics and a bender now with potentials applied) is then similarly captured into the Penning ion trap previously filled with cold $\mathrm{Be}^{+}$ions. Electrode switching and collisions with the xenon ions initially heat the $\mathrm{Be}^{+}$ions. This heating is associated with an increase in fluorescence rate. This laser scattering quickly re-cools the $\mathrm{Be}^{+}$ions, which cools the $\sim 400$ caught $\mathrm{Xe}$ ions. The mixed ion cloud cools, different charge-to-mass ratio ions separate with the cold highly charged Xe ions in the center of the trap and the $\mathrm{Be}^{+}$ions in an annulus that we image using scattered $313 \mathrm{~nm}$ light. A more detailed account of the procedure and results can be found in ref. [7]. Laser induced fluorescence from a magnetic dipole transition in the ground term of a suitable highly-charged ion can be potentially used to image the highly charged species in the trap[4]. 


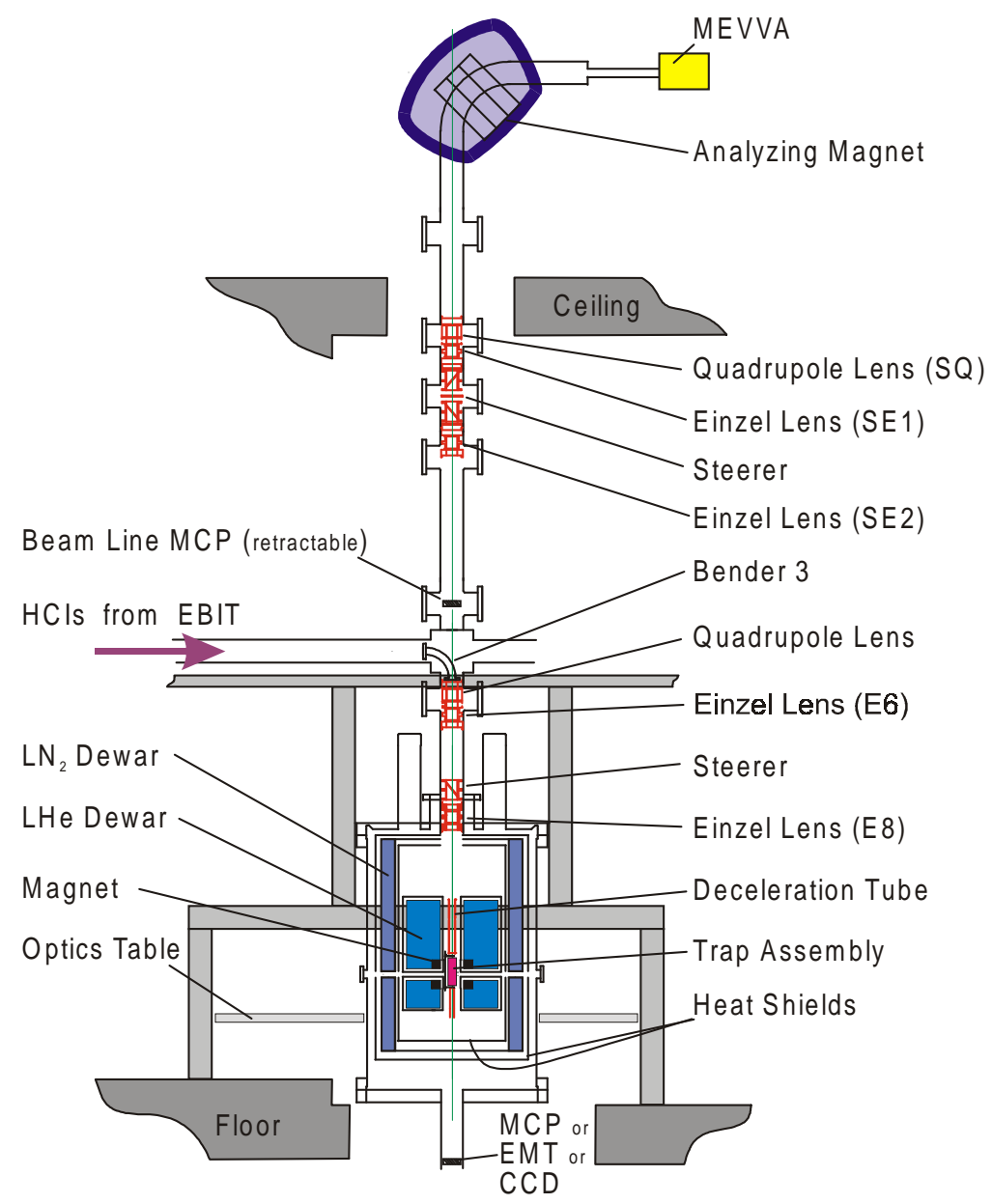

FIGURE 1. Setup to inject MeVVA ions into trap at LLNL. Magnet is used to separate charge states produced by MeVVA.

The shape of $\mathrm{Be}^{+}$fluorescent image can be used to determine the plasma parameters such as density and rotation rate. An ion species of interest can be excited by cyclotron resonance excitation (in the present trap configuration the frequencies associated with $\omega_{+}$, the modified cyclotron frequency is excited) resulting in heat transfer to the $\mathrm{Be}^{+}$ions and, typically, an increase in scatter of cooling laser light by the hotter $\mathrm{Be}^{+}$ions. Both the frequency and time response of this heating signal are of interest.

A preliminary study of cooling laser detuning (cooling power) with various radio frequency excitations (heating) of an ion cloud resulting from a $\mathrm{Be}^{+}-\mathrm{Xe}^{34+}$ capture shows interesting features (see figure 3). These signals may possibly indicate crystallization, some other configuration/coupling change between species or some systematic effect that is not understood. The laser tuning was monitored (using the fundamental of the doubled laser) against an $\mathrm{I}_{2}$ absorption cell for stability in frequency and amplitude. Similar signals have been assigned for small numbers of ions in a Paul trap[9]. Unfortunately, pump laser failure and other considerations prevented further studies. We wish to pursue similar studies at the new location of the experiment.

The trap contents, when warmer, can be examined with the tuned circuit used for electronic cooling as described above. The trap contents can also be destructively probed by releasing the ions into various charged particle detectors. 


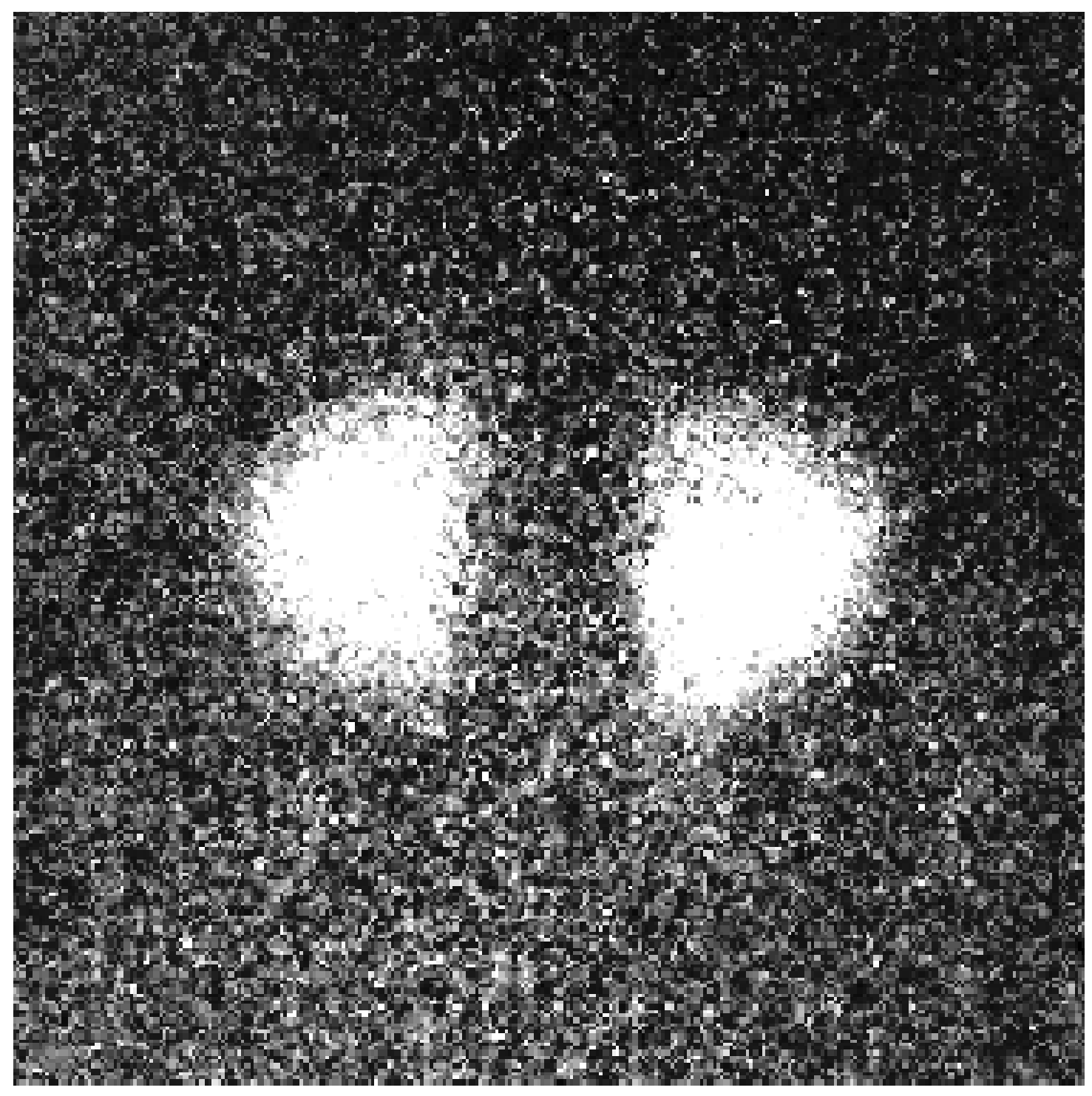

FIGURE 2. Image of $\mathrm{Be}^{+}$ion cloud containing $\mathrm{Xe}^{34+}$ and daughter charge exchange ions. B field is roughly vertical. With the cooling laser detuning and simulations it is estimated that the Xe ion temperature is approximately $1 \mathrm{~K}$. The image has been analyzed to give an $\mathrm{Be}^{+}$ion density $2.1610^{14} \mathrm{~m}^{-3}$ and a $\mathrm{Xe}^{34+}$ density of $6.410^{12} \mathrm{~m}^{-3}$. This corresponds to a $\Gamma$ value of about 577. 


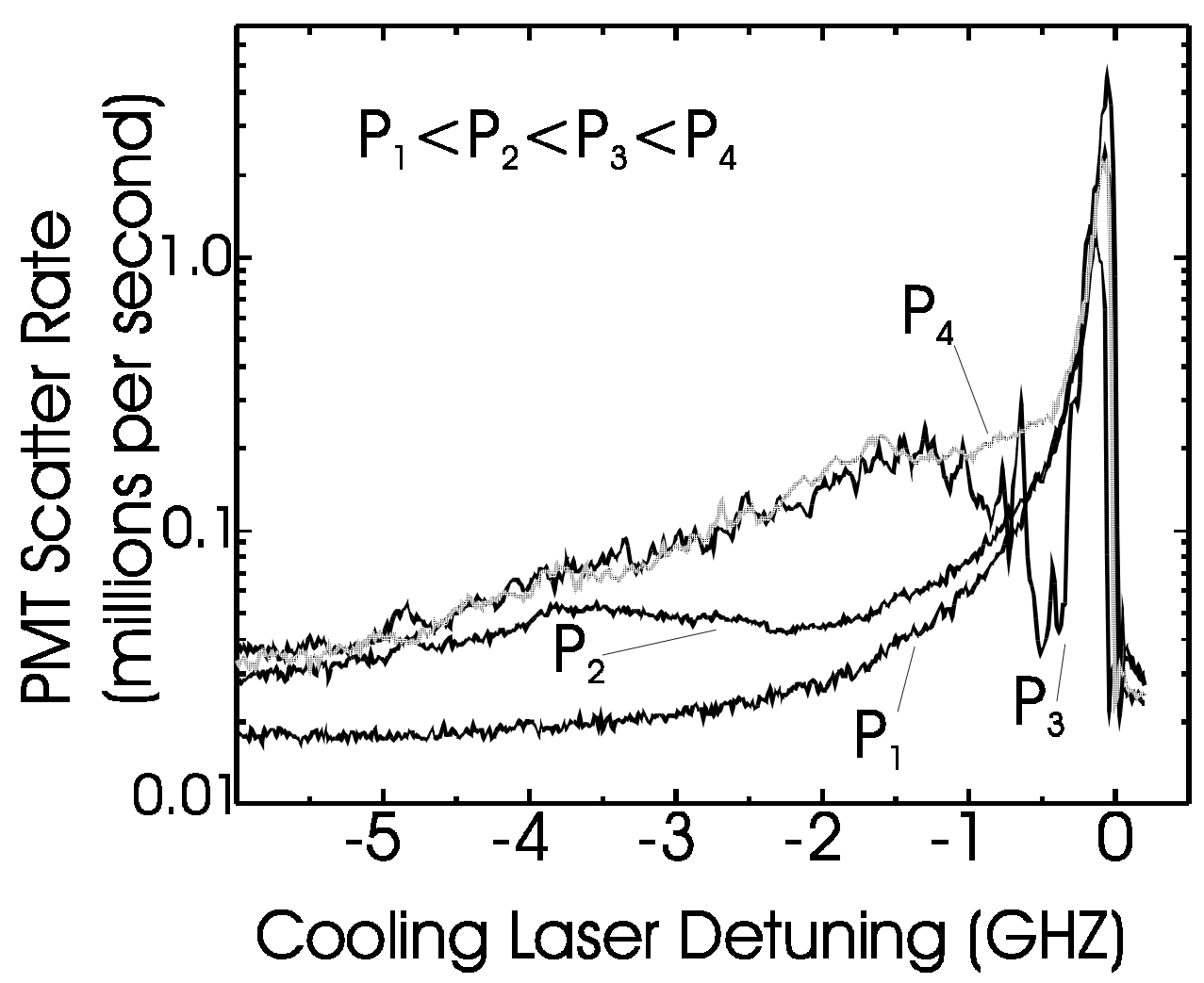

FIGURE 3. Cooling laser tuning curves. Main perpendicular cooling laser is used. $\mathrm{P}_{1}$ is base curve with no extra radio frequency heating applied. From other experiments the plasma temperature is about 1 Kelvin when the the detuning for the $\mathrm{P} 1$ curve is $200 \mathrm{MHz}$. The other curves have small amounts of radio frequency power applied near the Be cyclotron frequency. The trap contains $\mathrm{Be}^{+}, \mathrm{Xe}^{34+}$ and charge exchange daughter products. Plasma conditions are similar to that of Figure 2. The same ion cloud is used for all curves.

\section{SIMULATION}

A molecular dynamics code originally developed by E. L. Pollock, and used by H. DeWitt et al. to model strongly coupled plasmas [10] has been modified to run on personal computers. It was modified to exclusively calculate the interaction of two ion species confined in a Penning trap. Simple heating or cooling of each individual species was provided. 


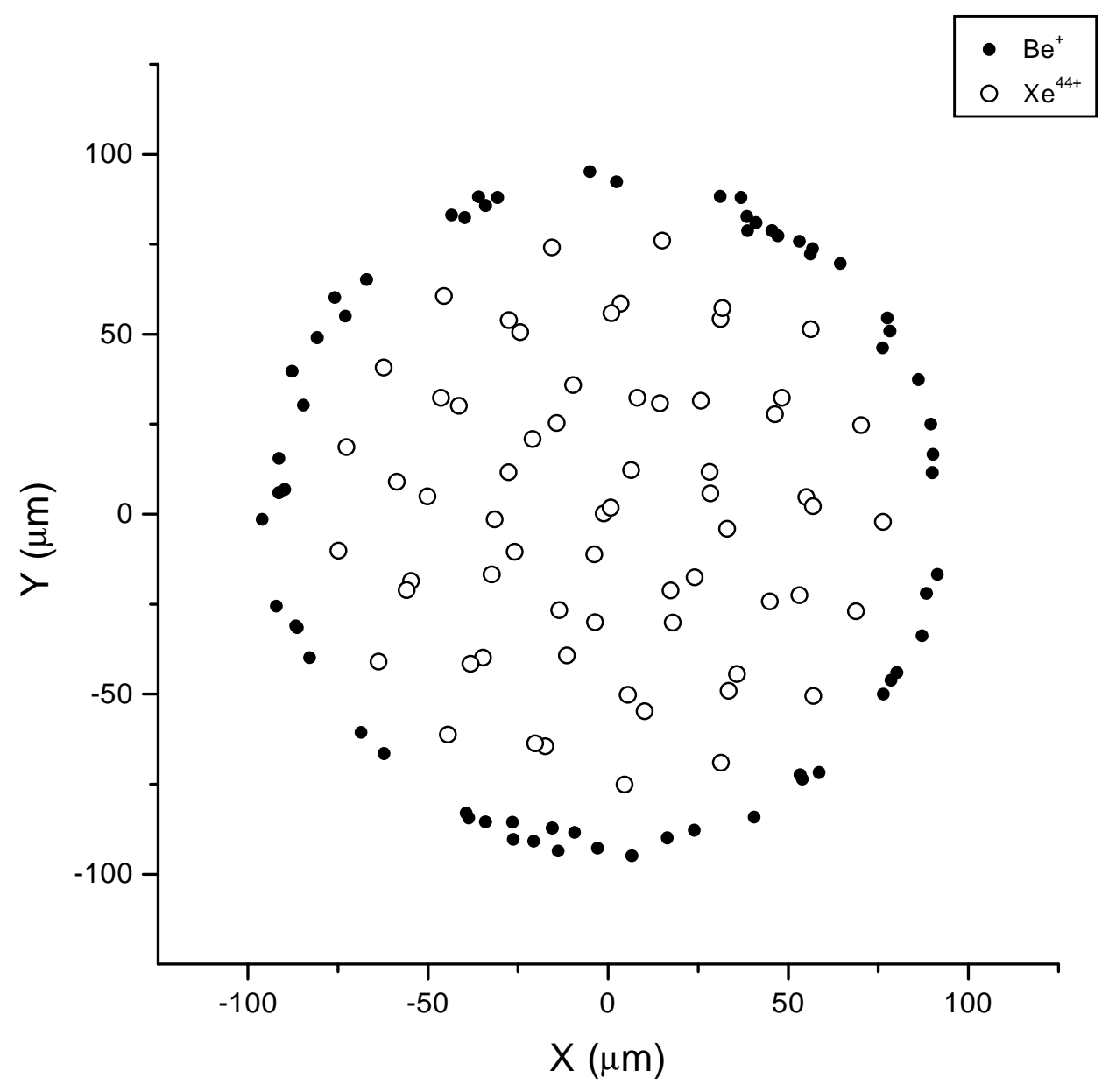

FIGURE 4. A "snapshot" $\mathrm{x}-\mathrm{y}$ projection of the cloud of $64 \mathrm{Be}^{+}$and $64 \mathrm{Xe}^{44+}$ ions used for heating rate calculations. This projection is for $0.5 \mathrm{Kelvin}$ axial cooling of the $\mathrm{Be}^{+}$ions and $1000 \mathrm{Kelvin} /\left(\right.$ particle $\bullet$ second) axial heating of the $\mathrm{Xe}^{44+}$ ions. The trap parameter is $\mathrm{q}=-2.0$ corresponding to about 540 Volts applied to our trap. The rotation frequency of the cloud is approximately $865 \mathrm{kHz}$.

Simulations of the motion of 5 to 64 multiply charged ions (experimental $\sim 100-500$ ) and 64 to 200 $\mathrm{Be}^{+}$(experimental $\sim 10^{5}$ ) ions were performed using fields, potentials, ion masses, and ion charges correspond to those used in the experimental work. Initially, the ions were arranged in a body-centered cubic lattice, with random assignment of initial locations of each species. The center of mass was placed at the trap center to simplify calculation. The ions were assigned pseudo-random initial velocities corresponding to an initial temperature, with the net linear momentum of the ensemble set to zero. The initial ion density, aspect ratio of the lattice, and ion rotation velocity were assigned. 


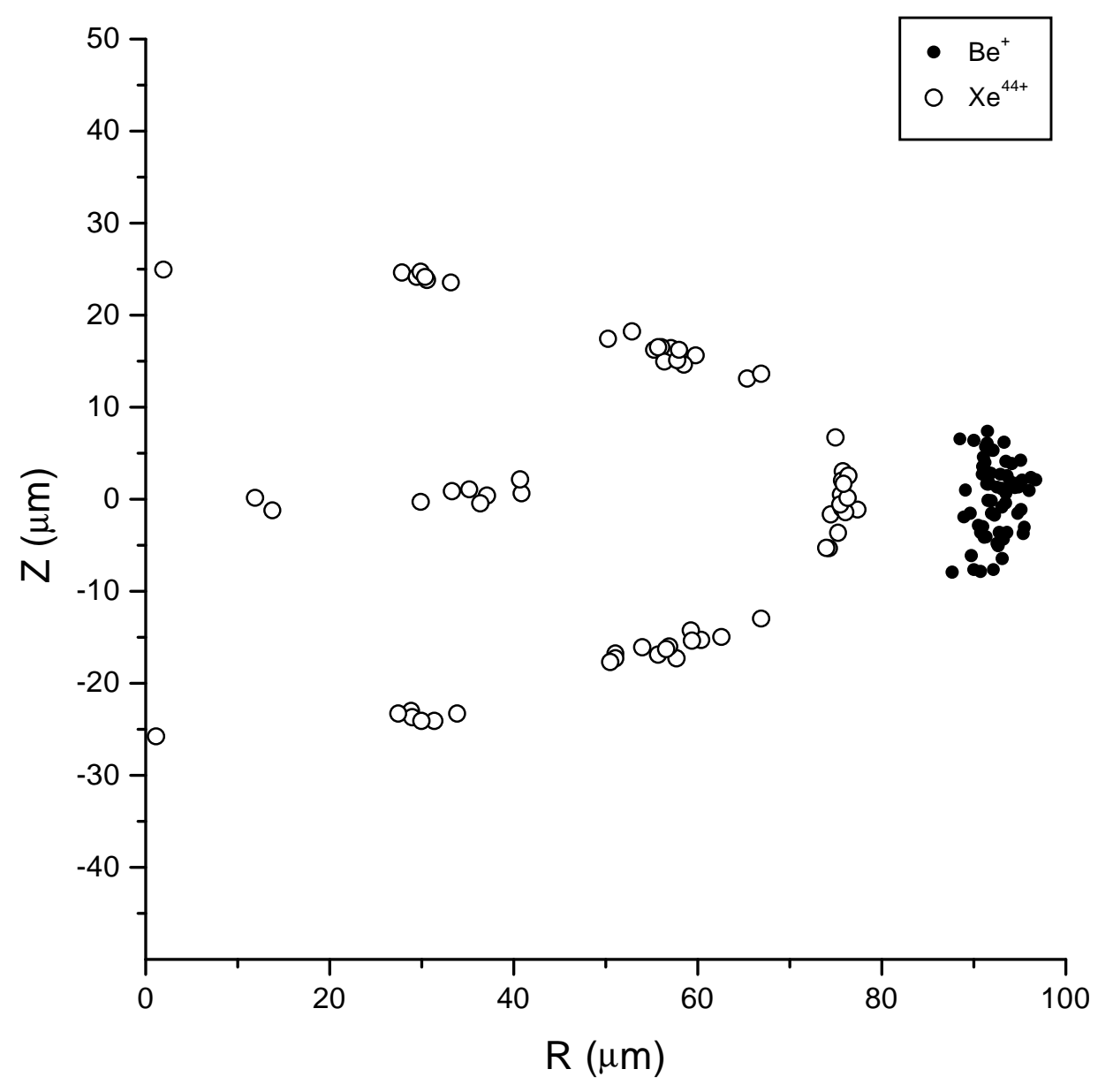

FIGURE 5. A "snapshot" cylindrical R-z projection of the ion cloud described in Figure4.

The electrostatic force between each pair of particles was calculated using Coulomb's Law between point charges. The electrostatic trap potential was modeled as an ideal hyperbolic potential, with the voltage applied between ring and end caps of a trap of specified size characterized by a single parameter $\mathrm{q}_{\text {trap. }}$. For the $9.34 \mathrm{~mm}$ scale length trap, $\mathrm{q}_{\text {trap }}=-2$ corresponds to about $540 \mathrm{~V}$. This approximates the voltage at which the axial oscillation of a $\mathrm{Be}^{+}$ion is resonant with the $2.5 \mathrm{MHz}$ tuned circuit. The value $\mathrm{q}_{\text {trap }}=-0.039$ corresponds to a shallow axial well, used experimentally for lower ion plasma rotation frequencies, resulting in greater interaction stability between the laser beam and the plasma. Dynamic features associated with ion confinement measurements, such as r.f. excitations, thermal bath coupling to tuned circuits, and image charges, were not modeled.

The equation of motion of the ions was integrated in the lab frame using the Verlet method [11], based on the Stoermer formula $\mathbf{r}_{i}(t+\tau)=2 \mathbf{r}_{i}(t)-\mathbf{r}_{i}(t-\tau)+\tau^{2} \mathbf{a}_{i}(t)+0\left(\tau^{4}\right)$. Here $\mathbf{a}_{i}$ is the acceleration of ion $\mathrm{i}$ due to the electric and magnetic forces of the trap, as well as the Coulomb forces. Since the trap symmetry axis is aligned with the uniform magnetic field $\mathrm{B}$, this equation is simplest to apply in the $\mathrm{z}$ coordinate. For the $\mathrm{x}$ and $\mathrm{y}$ radial coordinate, the time-centered velocity $\mathbf{v}_{\mathrm{i}}(\mathrm{t})=\left(\mathbf{r}_{\mathrm{i}}(\mathrm{t}+\tau)-\mathbf{r}_{\mathrm{i}}(\mathrm{t}-\tau)\right) / 2 \tau+$ $0\left(\tau^{3}\right)$ was used to evaluate the Lorentz force in the Stoermer formula and to get implicit expressions for $\mathrm{x}(\mathrm{t}+\tau)$ and $\mathrm{y}(\mathrm{t}+\tau)$ in terms of present acceleration, $\mathrm{x}$ and $\mathrm{y}$ position, and a single past time step of 
stored past $\mathrm{x}$ and $\mathrm{y}$ positions. A more sophisticated approach is described in the work of Spreiter and Walter, which we hope to incorporate in future simulations[12]. The parameter $\tau$ was chosen to be a fraction of the period of the initial plasma oscillation, so that it is also small enough to follow the largest cyclotron frequency of the simulation. It was found that small values of $\tau(\sim 30-300$ ps compared to $\sim 150 \mathrm{~ns} \mathrm{Be}^{+}$cyclotron period) resulted in a stable simulation start.

The mean squared axial velocity component (proportional to axial temperature $T_{z}$ of each species) is averaged for every other step. Every 1000 steps, an estimate of the rigid body rotation frequency was made, and in that reference frame, the mean squared radial velocity for each species (proportional to $\mathrm{T}_{\mathrm{r}}$ ) was calculated and recorded, along with the fixed frame kinetic energies and potential energies of the ions. When simulating cooling, if the running average axial temperature of an ion species exceeds the specified temperature by $3 \%$, the former z-axis positions of the species being "cooled" (proportional to axial velocity in a harmonic well) were re-scaled using a Taylor series expansion to approach the desired temperature. The other ion species could also be "heated" axially by a similar scaling, allowing a fixed amount of kinetic energy to be added on average to each ion. The temperature of the heated species can approach a steady state with the cooled species. Typically, the target temperature is lowered in steps as the system approaches quasi-steady state.

Major results of the simulation include observation of the onset of properties of a highly magnetized non-neutral plasma $[13,14]$. The multiply charged ions, with higher cyclotron frequencies, exhibited this effect at higher temperatures than the $\mathrm{Be}^{+}$ions, (ranging from $40 \mathrm{~K}$ to $2 \mathrm{~K}$ for $\mathrm{Xe}^{44+}$ ) depending on density (or equivalently cloud rotation frequency), as the "radial cyclotron temperature" decoupled at an exponential rate from the "axial temperature".

For example, a simulation of $64 \mathrm{Be}^{+}$ions and $64 \mathrm{Xe}^{44+}$ ion in a $\mathrm{q}_{\text {trap }}=-2.0$ well and with a cold rigid body rotation frequency of $865 \mathrm{kHz}$, this temperature was about 0.5 Kelvin. A snapshot of this simulation is shown in Figures 4 and 5. The ions are separated, as discussed below. However, the radial and axial motions remain coupled through the ion cloud rotation. The $\mathrm{Be}^{+}$ions were not significantly magnetized for simulated temperatures $>1$ Kelvin.

Typically, the multiply charged ions and $\mathrm{Be}^{+}$ions centrifugally separate, as Figures 3 and 4 show. This centrifugal separation occurs when the plasma rotates as a rigid body. The "pseudopotentials" associated with the plasma rotation for each species then differ, because of the different mass-to-charge ratios, causing the ions with higher mass to charge ratio (in this case $\mathrm{Be}^{+}$) to move radially outward and separate from the HCIs. The separation temperature was found to depend on the trap parameter $\mathrm{q}_{\text {trap }}$ and rotation frequency (bounds of which depend on $\mathrm{q}_{\text {trap }}$ ) as expected, although the current code (no spacial averaging / small numbers of particles) and mode of simulation (step down of set temperature) does not track the separation in detail. Simultaneously ordering of the HCIs can be seen, first as shells, then, at lower temperatures, rings of ions are apparent. The shell surfaces then have the characteristic triangular structure of 2D Coulomb systems [14,15].

Additional work involves the study of the effect of heating sources on the multiply charged ion temperatures given an experimentally measurable $\mathrm{Be}^{+}$temperature. From simulated motion and atomic data, spectroscopic lineshapes can be calculated and parameters for future experiments estimated. For example, $\mathrm{Ca}^{14+}$ has been caught and electronically cooled in our trap. Unfortunately, $\mathrm{Be}^{+}$laser cooling was not functioning at that time due to laser failure. Calculations are proceeding to model this system to optimize our procedure for a future experimental opportunity. 


\section{THE MOVE}

The Retrap facility has been moved to the Lawrence Berkeley National Laboratory. The trap is now sited on the roof of a target cave at the LBL 88" cyclotron[16]. The system is now undergoing preliminary testing with a MeVVA ion source. Several electron cyclotron resonance (ECR) ion sources will be available to continue work with highly charged ions. The laser capabilities needed for cooling and spectroscopy is to be reestablished in the near future. The cyclotron and associated ion source technology will allow the trapping and study of rare isotopes. The effect of charge state, for example, on various nuclear decay mechanisms can be studied.

\section{ACKNOWLEDGMENTS}

This work was performed under the auspices of the U.S. Department of Energy by the University of California Lawrence Livermore National Laboratory under Contract No. W-7405-ENG-48. Support of Texas A\&M authors through NSF grant PHY-9876899 and State of Texas ARP \#010366-0018-1997.

Thanks to Dr. G. Zwicknagel for pointing out the work of Spreiter and Walter.

\section{REFERENCES}

1. O'Neil, T.M., Physics Today 52, 24-30 (1999).

2. Chabrier, G., "Review on white dwarf cooling theory" in $11^{\text {th }}$ European Workshop on White Dwarfs, edited by S. -E. Solheim and E.G. Meistas, ASP Conference Series 169, San Francisco: Astronomy Society of the Pacific, 1999, p. 369.

3. www.whitedwarf.org

4. Church, D. A. et al, "RETRAP: An Ion Trap for Laser Spectroscopy of Highly-Charged Ions" in Trapped Charged Particles and Fundamental Physics, edited by D.H.E. Dubin and D. Schneider, AIP Conference Proceedings 457, New York: American Institute of Physics, 1999, pp. 235-241.

5. Schneider,D, et al., Rev. Sci. Instrum. 65, 3472 (1994).

6. Gruber, L., Cooling of Highly Charged Ions in a Penning Trap, UCRL-LR-138667, Lawrence Livermore National Laboratory, 2000.

7. Gruber, L. et al, PRL 86, 636-639 (2001).

8. Brewer,L.R. et al., Phys. Rev A57, 859 (1988).

9. Blumel, R. et al, Nature 334, 309-13 (1988). 
10. DeWitt, H.E et al, "Monte Carlo Simulation of the OCP Freezing Transition" in Strongly Coupled Plasma, edited by H. M. Van Horn and S. Ichimaru, Rochester: University of Rochester Press, 1993, p. 425.

11. Verlet, L., Phys. Rev 159, 98 (1967).

12. Spreiter, Q., and Walter, M., J. Comput. Phys. 152, 102-119 (1999).

13. Peurrung, A. J. et al, Int. J. Mass Spectrom. Ion Processes 157/158, 39 (1996).

14. Dubin, D.H.E. and O'Neil,T.M., RMP 71, 87 (1999).

15. Hasse, R.W. and Schiffer, J.P., Ann. of Phys. 203, 419 (1990).

16. http://www-nsd.lbl.gov/nsd/user88/ 


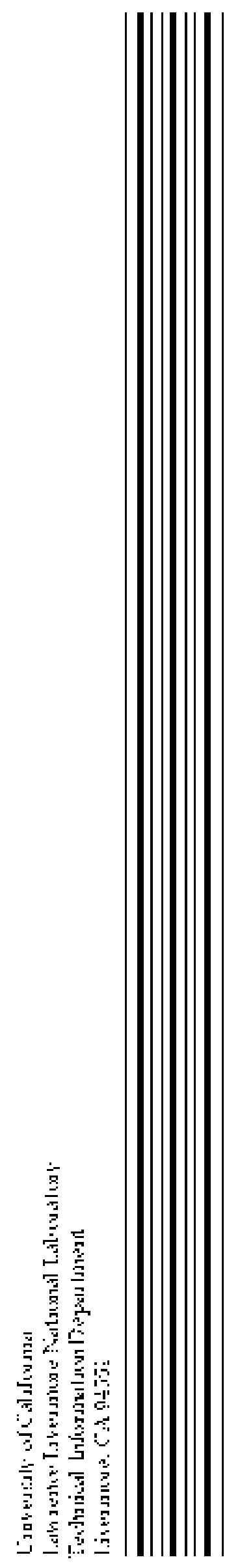

